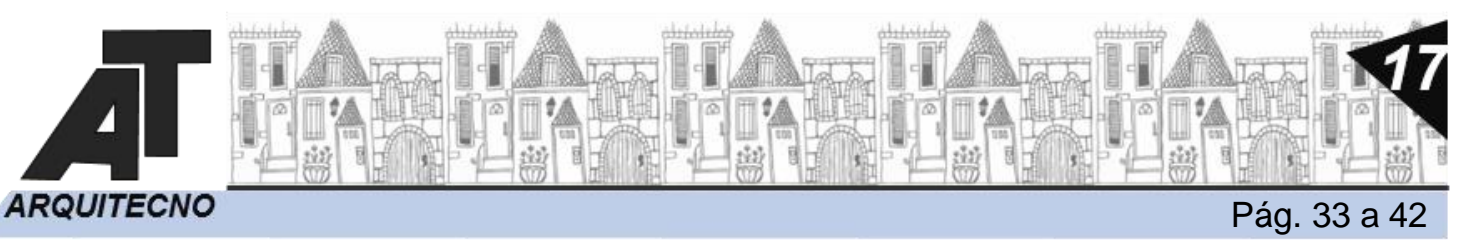

\title{
Gestión de la envolvente de una vivienda en verano: influencia del usuario sobre las condiciones de confort térmico interior
}

\author{
Management of a dwelling envelope in summer: \\ occupant influence on the indoor thermal comfort conditions
}

\author{
Soledad Andreoni Trentacoste ${ }^{1}$, Carolina Ganem ${ }^{2}$ \\ 1; 2 Instituto de Ambiente, Hábitat y Energía (INAHE), CONICET. Av. Ruiz Leal s/n, CP 5500, \\ Mendoza, Argentina.
}

sandreoni@mendoza-conicet.gob.ar

\section{RESUMEN}

El comportamiento del usuario en la búsqueda del confort térmico interior, principalmente en la interacción con la envolvente, es un parámetro que afecta el desempeño térmico de edificios residenciales. El objetivo del presente trabajo es cuantificar las variables que influyen en la gestión de ventanas de una vivienda en la ciudad de Mendoza. Se presentan los resultados del análisis estadístico de los datos de gestión de aberturas en una vivienda en condiciones reales de uso en verano, haciendo foco en los registros de apertura y cierre de ventanas. El modelo predictivo que considera la interacción de las variables de temperatura interior y exterior obtiene un $83 \%$ de observaciones exitosamente clasificadas. Las conclusiones permiten establecer parámetros de referencia para identificar perfiles de uso para su posterior implementación en programas de simulación. Haciendo posible que los resultados simulados en forma predictiva se aproximen al desempeño real edilicio.

\section{ABSTRACT}

In the search for indoor thermal comfort, occupant behaviour in terms of the interaction with the enclosure, is a parameter that affects the thermal performance of residential buildings. The objective of this work is to quantify the variables that influence the management of windows of a dwelling in the city of Mendoza. The results of the statistical analysis of windows management data in a dwelling under real conditions of use in summer are presented, focusing on window opening and closing records. The predictive model that considers the interaction of indoor and outdoor temperature variables obtains $83 \%$ of successfully classified observations. Conclusions allow to set reference parameters to identify occupant behaviour profiles for later implementation in simulation programs. Making it possible for predictively simulated results to approximate real building performance.

PALABRAS CLAVES: comportamiento del usuario, modelo estadístico, arquitectura residencial

KEY WORDS: occupant behaviour, statistical modelling, residential architecture

FECHA DE RECEPCIÓN: 3/2/2021 | FECHA DE ACEPTACIÓN: 012/5/2021

DOI: $\underline{\text { http://dx.doi.org/10.30972/arq.0174981 }}$ 
Gestión de la envolvente de una vivienda en verano:

influencia del usuario sobre las condiciones de confort térmico interior

Còssima Cornadó, Pilar Garcia-Almirall, Jordi Casals y Amaya Caballero

\section{METODOLOGÍA}

Se llevó a cabo una campaña de medición de temperatura y humedad en una vivienda caso de estudio bajo condiciones reales de uso en verano. Al mismo tiempo, se tomaron registros de la interacción de los usuarios con la envolvente, enfocados en la apertura y cierre de ventanas. Los datos obtenidos fueron analizados mediante técnicas de procesamiento de datos (data mining) con el objetivo de cuantificar el comportamiento de los ocupantes. Se analizó la relación entre las variables independientes y la probabilidad de apertura de ventanas. En base a la metodología propuesta por Fabi et al. (2012) se utilizaron modelos de regresión logística, contemplando que la predicción de la probabilidad de apertura de ventanas debe considerarse sobre la base de las ecuaciones utilizadas anteriormente para describir estadísticamente el comportamiento real de uso y gestión. Esto se debe a que factores como el clima local, las costumbres culturales, la tipología y estructura edilicias, juegan un rol fundamental en el comportamiento de las personas.

\section{DESARROLLO}

\section{Evaluación post-ocupación y registro de datos.}

El $87.5 \%$ de las viviendas de la región denominada Gran Mendoza son del tipo viviendas unifamiliares de baja altura (DEIE, 2019). Los datos analizados en este trabajo, fueron obtenidos mediante mediciones experimentales de temperatura y humedad en una vivienda urbana (fig. $1 \mathrm{y}$ 2) en la ciudad de Mendoza ( $32^{\circ} 40^{\prime} \mathrm{LS} ; 68^{\circ} 51^{\prime} \mathrm{LO}$ y $750 \mathrm{msnm}$ ), bajo condiciones reales de uso en verano. Caracterizándose el clima local por una gran amplitud térmica diaria y estacional, con una temperatura máxima media en verano de $32^{\circ} \mathrm{C}$ siendo la mínima media de $18^{\circ} \mathrm{C}$. Se colocaron tres sensores tipo HOBO U10 en el interior de la vivienda, en dos dormitorios de planta alta y en el estar comedor en planta baja, y un sensor en el exterior. Las características de la vivienda auditada se detallan en tabla 1.
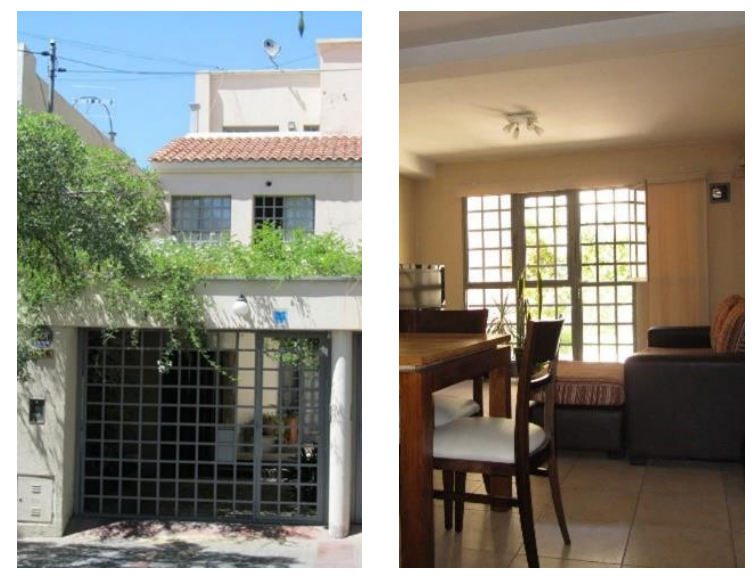

Figura 1 y 2: Fotografías de la fachada principal e interior de la vivienda. Fuente: Elaboración propia.

Se seleccionaron los datos medidos del ambiente principal de planta baja (estar comedor) y del exterior de la vivienda, durante 10 días en enero de 2017. Durante este periodo, los usuarios realizaron una gestión activa y comprometida de los elementos practicables de la envolvente, realizando la apertura de ventanas en horas de la noche para un correcto aprovechamiento de la ventilación natural. Esta última es una de las principales estrategias bioclimáticas recomendadas para lograr condiciones de confort interior con un menor consumo energético para el clima de la zona. Favoreciendo, en el caso de edificios con materiales de envolvente de alta inercia térmica, enfriar mediante convección la masa térmica durante la noche permitiéndole 


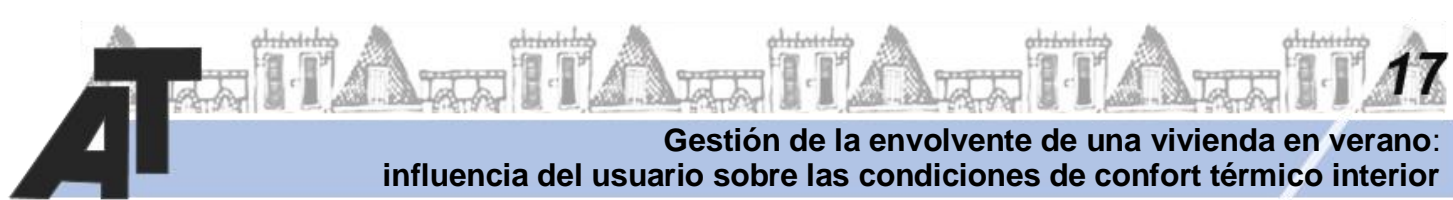

ARQUITECNO

Soledad Andreoni Trentacoste y Carolina Ganem

absorber el calor que pueda ingresar dentro del edificio durante el día, con solo un leve aumento de la temperatura interior (Givoni, 1992). Para el registro de uso y gestión se implementó la metodología de recolección de datos en forma de "diario" (Simone, Carpino, \& Gauthier, 2018), la cual consiste en planillas de auto completado con entradas estructuradas, también llamadas "encuestas de uso del tiempo" (TUS, por sus siglas en inglés). Esta metodología tiene la ventaja de ser comparable y replicable siempre que se utilice la misma estructura de planilla, su limitación es el requerimiento de compromiso por parte del sujeto entrevistado.

Tabla 1. Características constructivas de la vivienda.

\begin{tabular}{|c|c|c|c|}
\hline \multicolumn{4}{|c|}{ MATERIALES VIVIENDA } \\
\hline ELEMENTO & MATERIALES & $\begin{array}{c}\mathrm{U} \\
\left(\mathrm{W} / \mathrm{m} 2^{\circ} \mathrm{C}\right)\end{array}$ & $\begin{array}{l}\text { ESPESOR } \\
(\mathrm{cm})\end{array}$ \\
\hline \multirow{3}{*}{ MURO } & revoque ext. & 1.16 & 0.05 \\
\hline & ladrillon & 0.81 & 0.18 \\
\hline & revoque int & 1.16 & 0.03 \\
\hline \multirow[t]{2}{*}{ VENTANAS } & $\begin{array}{c}\text { chapa } \\
\text { plegada }\end{array}$ & 17 & 0.00071 \\
\hline & vidrio $4 \mathrm{~mm}$ & 0.9 & 0.004 \\
\hline \multirow{3}{*}{ TECHO } & teja cerámica & 0.76 & 0.06 \\
\hline & telgopor $5 \mathrm{~cm}$ & 0.03 & 0.05 \\
\hline & madera & 0.34 & 0.05 \\
\hline \multirow{4}{*}{ ENTREPISO } & $\begin{array}{l}\text { baldosa } \\
\text { cerámica }\end{array}$ & 0.7 & 0.03 \\
\hline & contrapiso & 1.63 & 0.15 \\
\hline & losa $\mathrm{H}^{\circ} \mathrm{A}^{\circ}$ & 1.74 & 0.20 \\
\hline & yeso aplicado & 0.48 & 0.025 \\
\hline \multirow{3}{*}{ PISO } & $\operatorname{los} a \mathrm{H}^{\circ} \mathrm{A}^{\circ}$ & 1.74 & 0.20 \\
\hline & contrapiso & 1.63 & 0.15 \\
\hline & $\begin{array}{l}\text { baldosa } \\
\text { cerámica }\end{array}$ & 0.7 & 0.03 \\
\hline \multirow[b]{2}{*}{ PISO PATIO } & contrapiso & 1.63 & 0.15 \\
\hline & $\begin{array}{c}\text { baldosa } \\
\text { cementicia }\end{array}$ & 1.15 & 0.15 \\
\hline
\end{tabular}

Fuente: Elaboración propia.

Los datos de monitoreo y registro recabados fueron: temperatura del aire interior y exterior, humedad relativa interior y exterior, registro de ocupación, gestión de ventanas y voto de confort interior (VF). Para evaluar el confort térmico y la satisfacción de los habitantes durante el día y la noche se utilizó el Estándar 55 de estudios ambientales de ASHRAE (ANSI/ASHRAE, 2013). En la siguiente tabla (2) se puede observar un resumen de los datos recabados:

Tabla 2. Resumen estadístico de datos de monitoreo y registro.

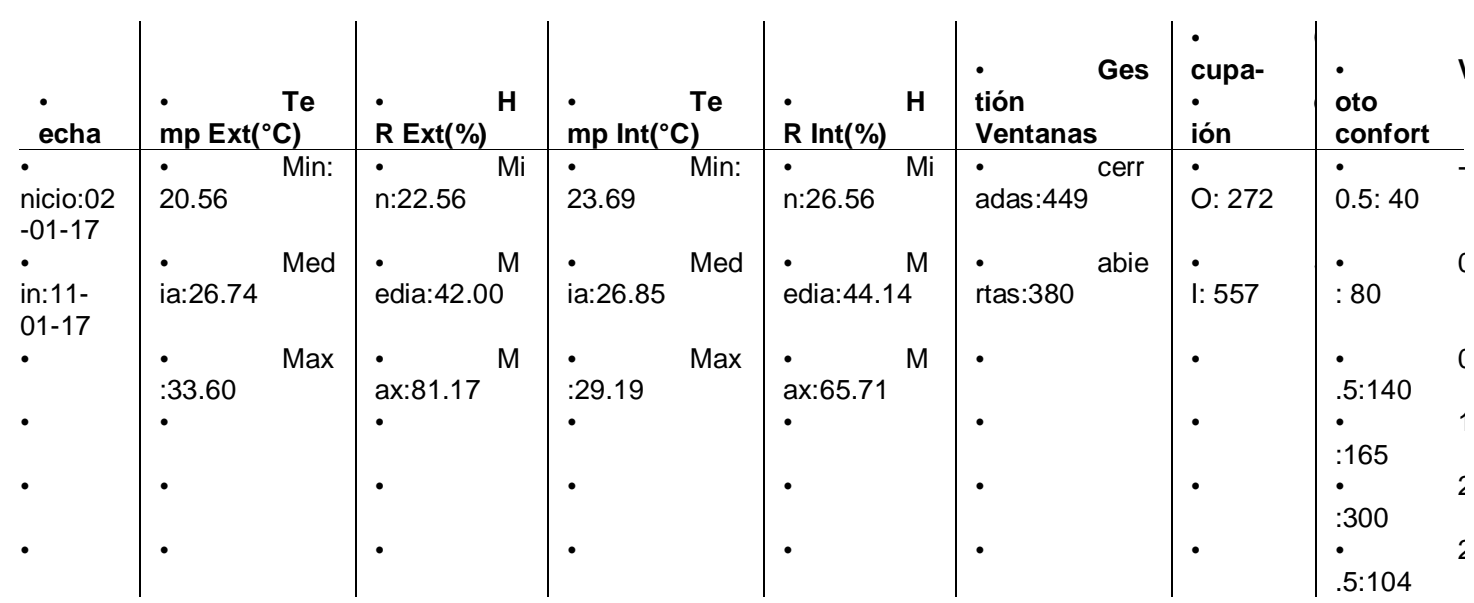

Fuente: Elaboración propia. 
Gestión de la envolvente de una vivienda en verano:

influencia del usuario sobre las condiciones de confort térmico interior

Còssima Cornadó, Pilar Garcia-Almirall, Jordi Casals y Amaya Caballero

\section{Procesamiento de datos}

Los datos auditados fueron analizados mediante técnicas de procesamiento de datos (data mining) por medio del software R (R Core Team, 2019). Se llevó a cabo un análisis estadístico comparativo de las posibles variables explicativas sobre la variable de respuesta discreta de apertura y cierre de ventanas. Se trabajó con modelos de regresión logística múltiple. Para el ajuste del modelo en $\mathrm{R}$ se especificó el parámetro family=binomial en la función $\mathrm{g} / \mathrm{m}(\mathbf{\text { (). }}$.

Para reducir la complejidad del modelo se consideraron solamente interacciones entre factores ambientales. Se descartó la variable de voto de confort como posible predictora, por ser intrínseca a la sensación térmica del usuario, y por estar directamente relacionada con la temperatura del ambiente interior, por lo tanto el estudio de esta última contempla la consideración de las condiciones de confort interior. También se descartó el análisis de la variable de ocupación como posible predictora, en este caso debido a que los registros confirman que el hecho de presencia o ausencia de los usuarios no tuvo influencia en el registro de apertura o cierre de ventanas, dejando en muchos casos las ventanas abiertas al salir de la vivienda.

La selección de variables predictivas del modelo se realizó utilizando el criterio de información Akaike (AIC), el mismo evalúa una a una las variables con el objetivo de encontrar la combinación con el menor valor de AIC (selección forward y backward). La literatura consultada (Andersen, et. al., 2013), recomienda esta metodología de valoración y selección de modelos cuando se trabaja con regresiones logísticas de datos observados y medidos de la realidad. Por último se comparó la potencialidad de predecir de cada modelo, utilizando procesos de validación cruzada, comparando los valores de sus tasas de clasificación de verdaderos positivos sobre falsos positivos (VP/FP) y verdaderos negativos sobre falsos negativos (VN/FN). Dicha clasificación denominada "matriz de confusión" se utiliza al predecir una variable categórica, la misma establece una comparación entre los datos predichos por el modelo y los datos del análisis del comportamiento real avaluado. Esto permite conocer el nivel de precisión del modelo a la hora de predecir un comportamiento futuro (Olson \& Delen, 2008).

\section{RESULTADOS}

Los resultados obtenidos de la auditoría realizada en un periodo de 10 días del mes de enero se pueden observar en el gráfico de la figura 3. Las temperaturas exteriores se muestran en línea continua de color negro, registrando una temperatura máxima de $33,60^{\circ} \mathrm{C}$ y una mínima de $20,56^{\circ} \mathrm{C}$. Las temperaturas interiores registradas para el mismo periodo se muestran en el mismo gráfico (fig.3) en línea de puntos con mapeo de color representativo de las mismas temperaturas. Se puede observar un buen desempeño térmico interior de la vivienda, ubicándose las temperaturas medias diarias de todo el periodo (línea continua en color naranja) por debajo de la máxima de confort recomendada para el clima local, de $28^{\circ} \mathrm{C}$. Sin embargo, las temperaturas máximas interiores registradas superan en los días más calurosos las máximas de confort establecidas, se puede asumir que una mejora en los elementos de aislación de la envolvente ayudaría a mejorar el desempeño térmico interior y evitar el sobrecalentamiento.

El periodo de análisis seleccionado resulta conveniente para la formulación de un modelo predictivo de gestión de la envolvente, ya que demuestra un comportamiento comprometido de los usuarios logrando el mejor desempeño térmico posible para la vivienda en estudio. 


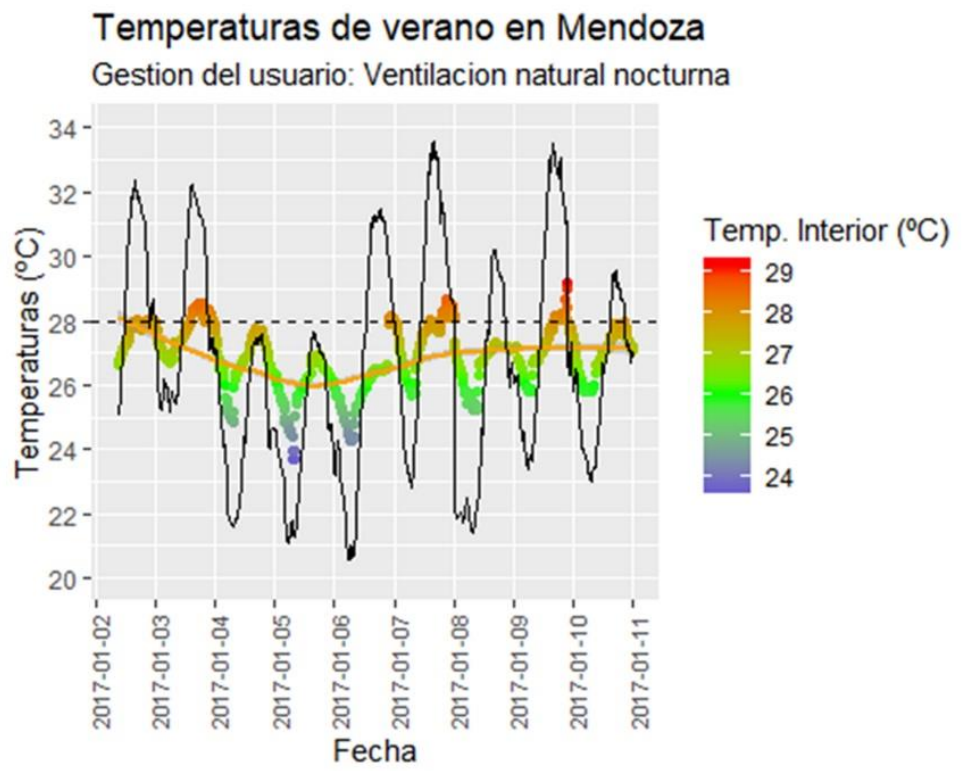

Figura 3: Temperaturas exteriores e interiores registradas, enero 2017. Fuente: Elaboración propia en R.

Las variables relacionadas a factores ambientales fueron analizadas para la formulación del modelo de regresión logística más preciso para predecir la respuesta de apertura y cierre de ventanas en condiciones de verano. Los resultados obtenidos de la selección de variables predictivas (tabla 3) muestran un menor valor de AIC para el modelo "dtH" que utiliza la interacción de variables de temperatura interior, temperatura exterior, humedad relativa interior y humedad relativa exterior. De los modelos analizados que contemplan solo los registros de temperatura, el modelo "dt" muestra el menor valor de AIC. Se descartan así los modelos que solo contemplan la influencia de la temperatura exterior o interior, demostrando que la percepción del usuario de la diferencia de temperatura entre el interior y el exterior de la vivienda motiva a la apertura o cierre de ventanas para conseguir mejores condiciones de confort interior.

Tabla 3. Resultado selección de variables predictivas AIC.

\begin{tabular}{|c|c|c|c|c|c|}
\hline - & & - & df & • & AIC \\
\hline$\bullet$ & modelo_text & $\bullet$ & 2 & - & 580.7192 \\
\hline - & modelo_tin & • & 2 & - & 657.3022 \\
\hline - & modelo_dt & $\bullet$ & 4 & - & 561.2358 \\
\hline - & modelo_dtH & • & 16 & - & 264.8814 \\
\hline
\end{tabular}

Si bien el modelo "dtH" muestra un menor valor de AIC que el modelo "dt", al compararlos por medio de procesos de validación cruzada la potencialidad de predecir la respuesta de apertura y cierre de ventanas del modelo "dtH" es menor.

En las figura 4 y 5 se pueden observar las tablas de clasificación de VP, FN, FP y VN de ambos modelos. La tasa de clasificación se maximizó para ambos casos, obteniendo para el modelo "dt" un punto de corte de 0.40 logrando así una tasa de clasificación de $83 \%$. Por el otro lado, para el modelo "dtH" se maximizó la tasa de clasificación en un punto de corte de 0.53 , obteniendo una tasa de clasificación de 78\%. 

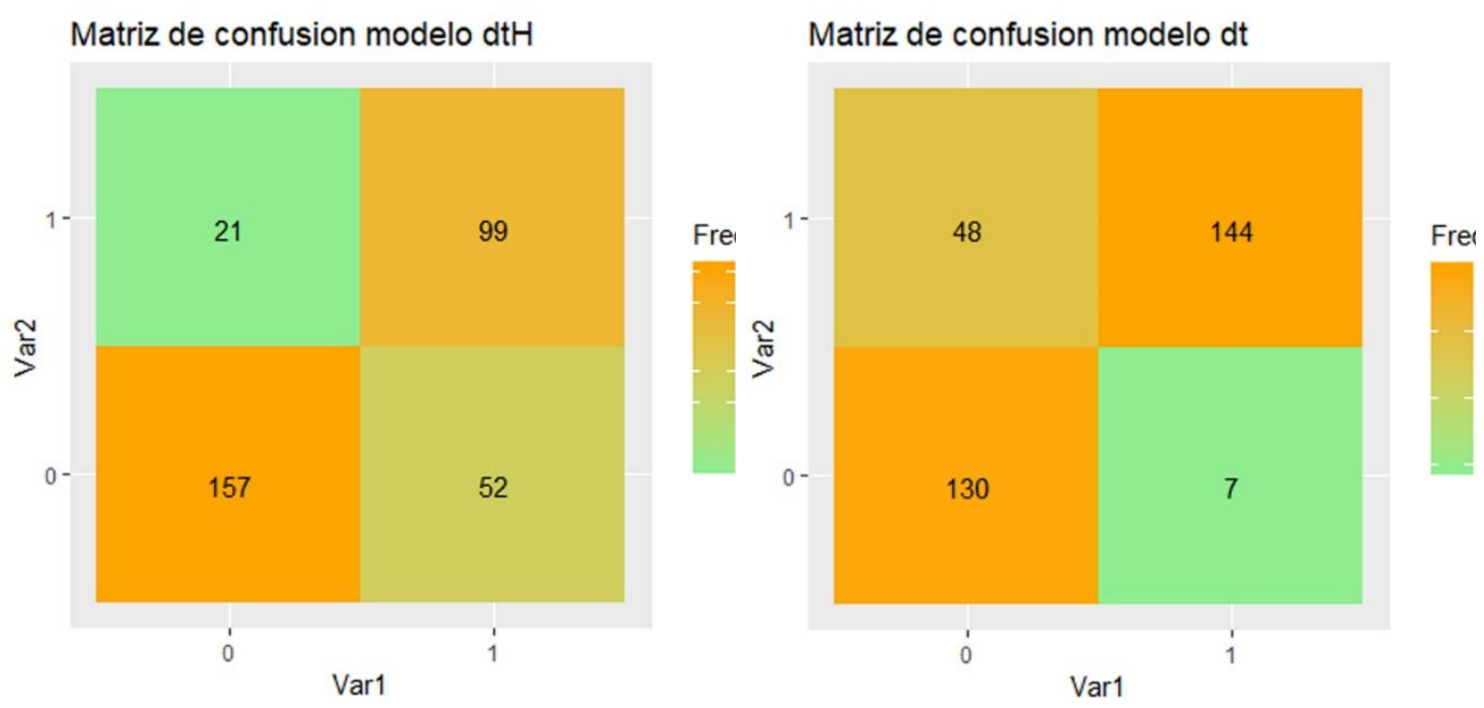

Figuras 4 y 5: Tablas de clasificación de VP/FP, VN/FN. Fuente: Elaboración propia en R.

Los resultados muestran, por lo tanto, que la humedad relativa interior y exterior influye en el comportamiento del usuario, pero no resulta favorable para predecir con mayor precisión el mismo. Siendo la interacción entre temperatura interior y exterior el modelo más conveniente para la predicción de la gestión de los elementos practicables de la envolvente en viviendas de la ciudad de Mendoza. El modelo "dt" considera que a mayor diferencia negativa (menor temperatura exterior que interior) aumenta la probabilidad de apertura de ventanas, dicha probabilidad disminuye a medida que la diferencia de temperatura se invierte (figura 6).

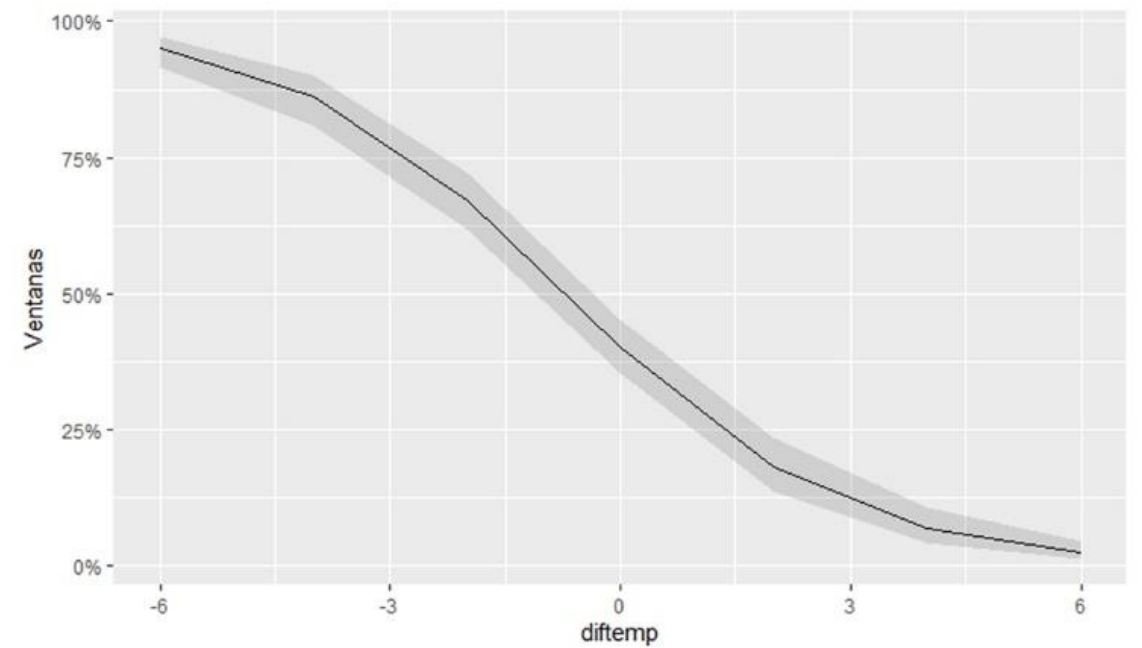

Figura 6: Gráfico del modelo de predicción de apertura de ventanas. Fuente: Elaboración propia en R.

El modelo "dt" resulta ser el más apropiado para el estudio de la predicción de respuesta de apertura y cierre de ventanas, aplicando como criterio la preferencia de obtener un menor error en la valoración de apertura de ventanas, por considerarla la acción de influencia más directa en la modificación de las condiciones de confort interior. 


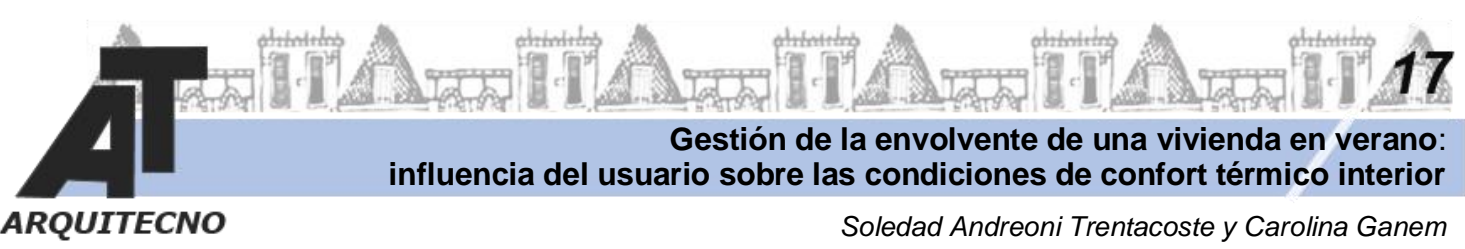

\section{CONCLUSIONES}

En base al monitoreo experimental de una vivienda caso de estudio, bajo condiciones reales de uso en verano en la ciudad de Mendoza, se formularon dos posibles modelos para la predicción de acciones de gestión de ventanas.

La interacción entre las variables de temperatura interior, temperatura exterior, humedad relativa interior y humedad relativa exterior resulto ser el modelo más representativo de la influencia real que provoca la gestión de apertura y cierre de ventanas en viviendas de la ciudad de Mendoza. Sin embargo, al evaluar la capacidad predictiva del modelo, la interacción de temperatura interior y exterior resulto tener más observaciones exitosamente clasificadas $(83 \%$ de las observaciones), mejorando de forma significativa al modelo anterior en la clasificación de eventos de apertura de ventanas. Se prefiere este modelo por priorizar un menor error en la predicción de eventos de apertura por sobre los de cierre de ventanas, debido a que el flujo de aire provocado por la ventilación cruzada impacta en forma inmediata en las condiciones de confort interiores.

Los resultados del presente estudio, demuestran que el comportamiento del usuario es un aspecto relevante en la valoración del desempeño térmico de viviendas urbanas en localidades de clima árido templado continental. El análisis estadístico realizado permite aproximarse a la predicción de la variable de respuesta de apertura y cierre de ventanas para el logro del confort interior, determinando a priori la importancia de considerar la interacción de ambas variables medidas de temperatura, tanto interior como exterior.

Futuros estudios continuarán la aplicación del modelo propuesto en modelos de simulación edilicia, contemplando el estudio de otros aspectos del comportamiento del usuario, con el fin de representar de forma más precisa la realidad del desempeño térmico edilicio en programas de simulación. 
Gestión de la envolvente de una vivienda en verano:

influencia del usuario sobre las condiciones de confort térmico interior

Còssima Cornadó, Pilar Garcia-Almirall, Jordi Casals y Amaya Caballero

ARQUITECNO

\section{BIBLIOGRAFÍA}

Andersen, R., Fabi, V., Toftum, J., Corgnati, S. P., \& Olesen, B. W. (2013). Window opening behaviour modelled from measurements in Danish dwellings. Building and Environment, 69, 101-113. https://doi.org/10.1016/j.buildenv.2013.07.005

Andreoni Trentacoste, S. E., \& Ganem Karlen, C. (2017). Influencia del uso y gestión de la envolvente en el comportamiento térmico de verano de una vivienda en la ciudad de Mendoza, Argentina. Revista Hábitat Sustentable, 7(2), 64-75. https://doi.org/10.22320/07190700.2017.07.02.06

ANSI/ASHRAE. (2013). Standard 55-2013. Thermal Environmental Conditions for Human Occupancy. Atlanta.

Arrieta, G. M. (2020). Valoraciones subjetivas y condiciones objetivas de confort térmico en viviendas. Arquitecno, 16(16), 57. https://doi.org/10.30972/arq.0164553

Clevenger, C. M., \& Haymaker, J. (2006). The impact of the building occupant on energy modeling simulations. In Joint International Conference on Computing and Decision Making in Civil and Building Engineering (pp. 1-10). Montreal, Canada,.

DEIE. (2019). Encuesta condiciones de vida. Informe anual. Mendoza.

Fabi, V., Andersen, R. V., Corgnati, S. P., \& Olesen, B. W. (2013). A methodology for modelling energy-related human behaviour: Application to window opening behaviour in residential buildings. Building Simulation, 6(4), 415-427. https://doi.org/10.1007/s12273-013-0119-6

Ganem, C., Esteves, A., \& Coch, H. (2005). El rol de la envolvente en la rehabilitación ambiental. Propuestas de Verano para viviendas tipo Chalet en Mendoza. Avances En Energías Renovables y Medio Ambiente, 9, 49-54.

Givoni, B. (1992). Comfort, climate analysis and building design guidelines. Energy \& Buildings, $18,11-23$.

Nicol, J. F. (2001). Characterisin occupant behaivour in buildings: towards a stochastic model of occupant ue of windows, lights, blinds heaters and fans. In Seventh International IBPSA Conference (pp. 1073-1078). Rio de Janeiro, Brazil: IBPSA.

Olson, D. L., \& Delen, D. (2008). Advanced data mining techniques. (1 Edition). Berlin: Springer.

Ouf, M. M., Gunay, H. B., \& O'Brien, W. (2019). A method to generate design-sensitive occupantrelated schedules for building performance simulations. Science and Technology for the Built Environment, 25(2), 221-232. https://doi.org/10.1080/23744731.2018.1514855

R Core Team. (2019). R: A language and environment for statistical computing. R Foundation for Statistical Computing. Vienna, Austria. Retrieved from https://www.r-project.org/

Rijal, H. B., Tuohy, P., Humphreys, M. A., Nicol, J. F., Samuel, A., \& Clarke, J. (2007). Using results from field surveys to predict the effect of open windows on thermal comfort and energy use in buildings. Energy and Buildings, 39(7), 823-836. https://doi.org/10.1016/j.enbuild.2007.02.003

Simone, M. De, Carpino, C., \& Gauthier, S. (2018). Reference procedures for obtaining occupancy profiles in residential buildings.

Wagner, A., \& O'Brien, W. (2018). Exploring Occupant Behavior in Buildings. (A. Wagner, W. O'Brien, \& B. Dong, Eds.). Switzerland: Springer International Publishing AG. https://doi.org/10.1007/978-3-319-61464-9

Yan, D., O'Brien, W., Hong, T., Feng, X., Burak Gunay, H., Tahmasebi, F., \& Mahdavi, A. (2015). Occupant behavior modeling for building performance simulation: Current state and future challenges. Energy and Buildings, 107, 264-278. https://doi.org/10.1016/j.enbuild.2015.08.032 

influencia del usuario sobre las condiciones de confort térmico interior 